\title{
Fully printed high performance humidity sensors based on two-dimensional materials
}

DOI:

10.1039/C7NR08115D

Document Version

Accepted author manuscript

Link to publication record in Manchester Research Explorer

\section{Citation for published version (APA):}

He, P., Brent, J., Ding, H., Yang, J., Lewis, D., O'Brien, P., \& Derby, B. (2018). Fully printed high performance humidity sensors based on two-dimensional materials. Nanoscale, 10(12), 5599-5606.

https://doi.org/10.1039/C7NR08115D

\section{Published in:}

Nanoscale

\section{Citing this paper}

Please note that where the full-text provided on Manchester Research Explorer is the Author Accepted Manuscript or Proof version this may differ from the final Published version. If citing, it is advised that you check and use the publisher's definitive version.

\section{General rights}

Copyright and moral rights for the publications made accessible in the Research Explorer are retained by the authors and/or other copyright owners and it is a condition of accessing publications that users recognise and abide by the legal requirements associated with these rights.

\section{Takedown policy}

If you believe that this document breaches copyright please refer to the University of Manchester's Takedown Procedures [http://man.ac.uk/04Y6Bo] or contact uml.scholarlycommunications@manchester.ac.uk providing relevant details, so we can investigate your claim.

\section{OPEN ACCESS}



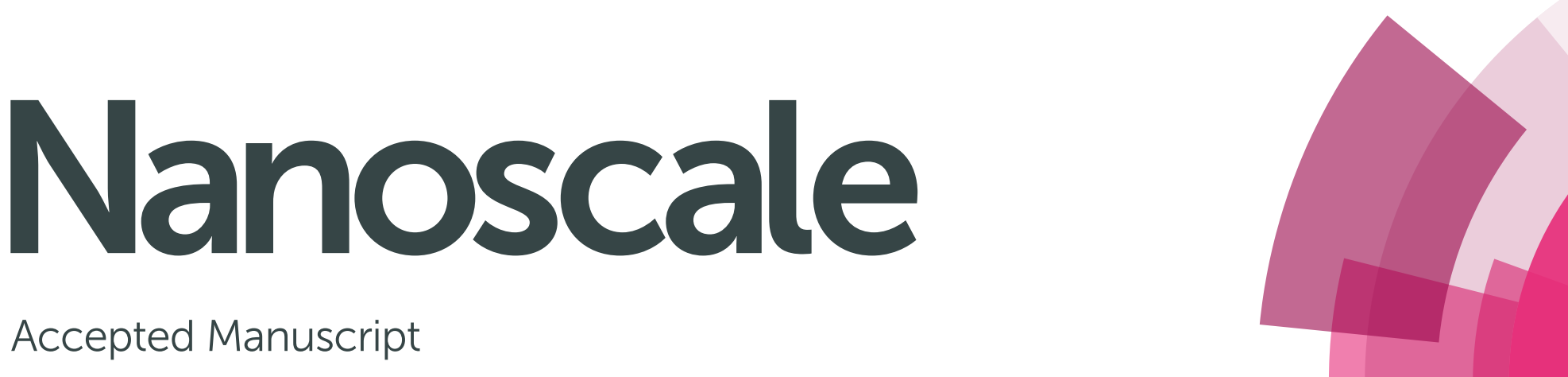

\section{Accepted Manuscript}

This article can be cited before page numbers have been issued, to do this please use: P. He, J. R. Brent, H. Ding, J. Yang, D. J. Lewis, P. O'Brien and B. Derby, Nanoscale, 2018, DOI: 10.1039/C7NR08115D.

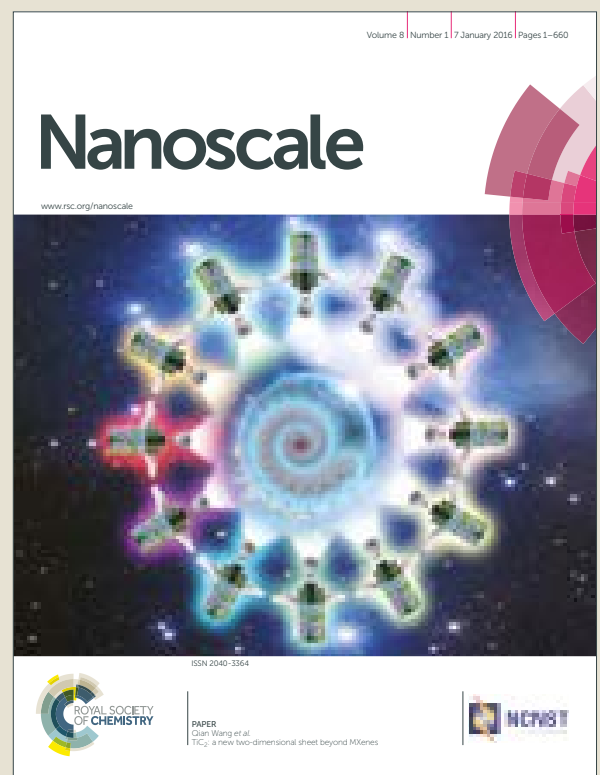

This is an Accepted Manuscript, which has been through the Royal Society of Chemistry peer review process and has been accepted for publication.

Accepted Manuscripts are published online shortly after acceptance, before technical editing, formatting and proof reading. Using this free service, authors can make their results available to the community, in citable form, before we publish the edited article. We will replace this Accepted Manuscript with the edited and formatted Advance Article as soon as it is available.

You can find more information about Accepted Manuscripts in the author guidelines.

Please note that technical editing may introduce minor changes to the text and/or graphics, which may alter content. The journal's standard Terms \& Conditions and the ethical guidelines, outlined in our author and reviewer resource centre, still apply. In no event shall the Royal Society of Chemistry be held responsible for any errors or omissions in this Accepted Manuscript or any consequences arising from the use of any information it contains. 


\title{
Journal Name
}

\section{ARTICLE}

\section{Fully printed high performance humidity sensors based on two- dimensional materials}

Received 00th January 20xx, Accepted 00th January 20xx

DOI: $10.1039 / \times 0 \times x 00000 x$

www.rsc.org/

\author{
P. He ${ }^{\text {, }}$ J. R. Brent ${ }^{\text {a }}$, H. Ding ${ }^{\text {a }}$, J. Yang ${ }^{\text {, }}$ D. J. Lewis ${ }^{a}$, P. O. Brien ${ }^{b}$ and B. Derby *a
}

Fully printed humidity sensors based on two dimensional (2D) materials are described. Monolayer graphene oxide (GO) and few-layered black phosphorus (BP) flakes were dispersed in low boiling point solvents suitable for inkjet printing. The humidity sensors were fabricated by printing of GO and BP sensing layers on printed silver nanoparticle electrodes. The electrical response of the $\mathrm{GO}$ and BP sensors to humidity levels ranging from 11 to $97 \%$ relative humidity, which revealed high capacitance sensitivity of $4.45 \times 10^{4}$ times for the GO sensor and $5.08 \times 10^{3}$ times for the BP sensor at $10 \mathrm{~Hz}$ operation frequency. Response/recovery times of the GO and BP sensor were measured to be $2.7 / 4.6 \mathrm{~s}$ and $4.7 / 3.0 \mathrm{~s}$ respectively. These sensors also showed sensitive and fast response to a proximal human fingertip, giving potential applications in contactless switching.

\section{Introduction}

Humidity sensors are important for monitoring, detecting and controlling environmental humidity levels, with applications in a number of areas including: indoor/outdoor climate control, the semiconductor and food processing industries, for medical applications, and for monitoring human activity. ${ }^{1,2}$ Over the decades, a range of materials have been used in humidity sensors, including organic, inorganic and hybrid materials. ${ }^{3}$ Generally, an ideal humidity sensor material requires high sensitivity, a wide humidity detection range, a quick response and short recovery time, as well as the potential for inexpensive large scale fabrication. ${ }^{5}$ Humidity responsiveness normally relies on the total area of the sensing material exposed to moisture molecules; thus a range of different humidity sensitive materials with large specific surface areas have been developed in recent years, including: metal oxide nanorods or nanotubes, ${ }^{6,7}$ semiconductor nanoparticles or nanowires, ${ }^{9-11}$ and carbon nanotubes. ${ }^{12,} 13$ These materials have shown improvements in the sensitivity. However, for applications requiring fast sensor response and rapid recovery time, new humidity sensing materials are still required.

Recently, two-dimensional (2D) nanomaterials with layered structures have been widely studied for sensor applications because of their high specific surface area. ${ }^{14}$ Graphene, the first member of the $2 \mathrm{D}$ materials family to be isolated, is a two-dimensional material of one carbon atom thickness that has great potential for ultrasensitive detection because of its high surface area and exceptional electrical properties. ${ }^{15}$ Highly sensitive gas sensors based

\footnotetext{
a. School of Materials, University of Manchester, Oxford Road, M13 9PL, U.K. E-mail:Brian.Derby@manchester.ac.uk

b. School of Chemistry, University of Manchester, Oxford Road, M13 9PL, U.K. + Footnotes relating to the title and/or authors should appear here.

Electronic Supplementary Information (ESI) available: [details of any supplementary information available should be included here. See DOI: 10.1039/x0xx00000x
}

on graphene have been widely investigated. ${ }^{20}$ However, pristine graphene shows low sensitivity for moisture detection due to its hydrophobicity and high intrinsic electrical conductivity. ${ }^{21}$

Graphene oxide (GO), is a highly promising humidity sensing material. $8,23 \mathrm{GO}$ is a graphene derivative based on the $2 \mathrm{D}$ honeycomb structure of $s p^{2}$ carbon atoms, but containing numerous hydroxyl, carboxyl, and epoxy groups, which modify the chemical bonding and planarity of the 2D graphene sheet, the latter by introducing tetrahedral $s p^{3}$ carbon centres. ${ }^{26}$ These functional groups can interact with oxophilic water molecules and generate protons, leading to a decrease in electrical impedance. Although various humidity sensors based on GO have been investigated in the last several years, ${ }^{4}, 8$ the sensitivity of $\mathrm{GO}$ sensors still needs improvement. Recent experimental results suggest that the properties of GO-based humidity sensors can be improved by using larger size GO sheets; this should increase the effective proton transport length, because sheet-to-sheet junctions or boundaries will block the proton transport path. ${ }^{28}$ However $\mathrm{GO}$ also shows a similar response to the presence of $\mathrm{NH}_{3}$ and $\mathrm{NO}_{2} .{ }^{29}$ Other $2 \mathrm{D}$ materials, such as transition metal dichalcogenides (TMDCs) ${ }^{25}$ and black phosphorus $(\mathrm{BP})^{30}$ also show potential for high performance humidity sensing applications. BP has been reported as responding to humidity yet showing no or only slight response to $\mathrm{H}_{2}, \mathrm{O}_{2} \mathrm{CO}_{2}{ }^{30}$ and an increase in resistivity on exposure to $\mathrm{NH}_{3},{ }^{31}$ hence suggesting a better performance as a humidity sensor. However, these previous studies using BP have not used scalable manufacturing methods to fabricate the sensors.

2D layered sheets of graphene family materials in particular GO, can be readily produced in large quantity by liquid-phase exfoliation to form stable 2D material suspensions in various solvents that can subsequently be used as functional inks. ${ }^{32}, 33$ This makes them promising for large scale and low-cost manufacture through solution processes, such as inkjet and other printing routes. Hu et al. demonstrated a BP ink formulation for inkjet printing of optoelectronic devices. ${ }^{34}$ However, they used a two-step process for 


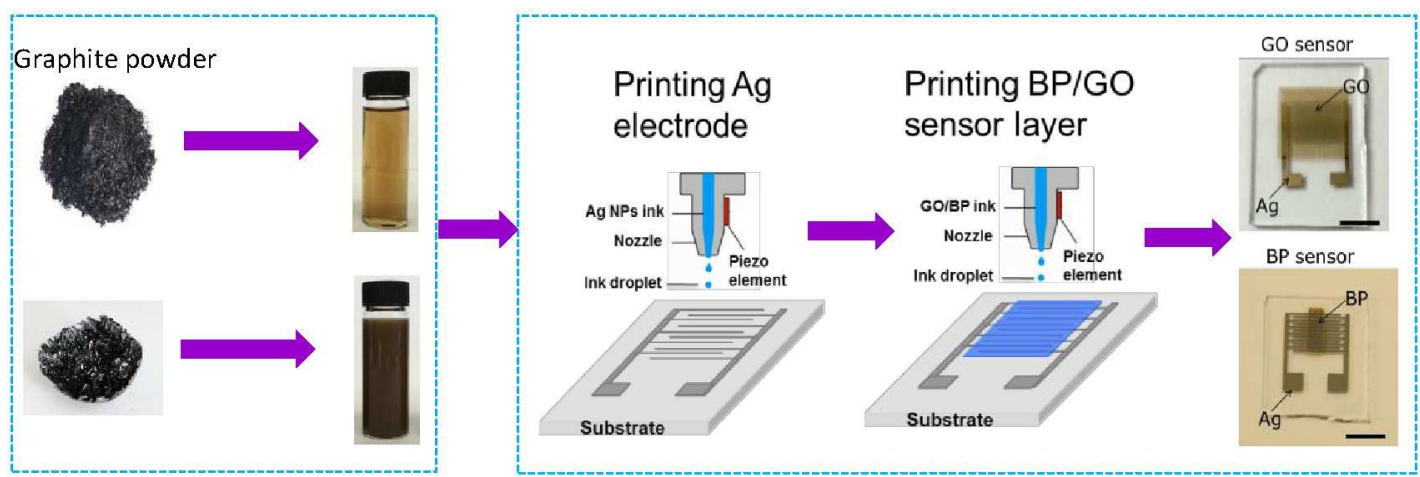

Fig. 1 Schematic representation of the fabricating process of sensor devices: (a) ink preparation and (b) inkjet printing of sensor devices. The scale bars are $5 \mathrm{~mm}$.

exfoliation and solvent exchange prior to ink preparation. Thus further optimisation of BP inks is required.

Here, we present a study of inkjet printed, high performance, humidity sensors using $\mathrm{GO}$ and few-layer $\mathrm{BP}$ as the sensing material combined with silver nanoparticle based electrodes. The GO sheets were dispersed in water, which showed a mean lateral size of $\sim 36$ $\mu \mathrm{m}$ and up to $\sim 200 \mu \mathrm{m}$ observed. The BP ink was prepared by exfoliating BP in a low boiling point solvent, acetonitrile (boiling point of 82 으). This ink preparation process, which uses low boiling point solvents, is more suitable for large-scale manufacturing application for printed electronics.

\section{Experimental method}

\section{Preparation of GO and BP inks}

GO was prepared by the modified Hummers method described previously. ${ }^{35}, 36$ Briefly, graphite powder (1.5 g, grade 9842, Graphexel Ltd., Epping, UK) was mixed with sulfuric acid ( $200 \mathrm{ml}, 17.7$ mol, Fisher Scientific, Loughborough, UK) while stirring and cooling in an ice bath. Subsequently, Potassium permanganate $\left(\mathrm{KMnO}_{4}, 1.5\right.$ g, (9.49 mmol) Sigma-Aldrich, Gillingham, UK) was slowly added over 20 minutes. The reaction was maintained for $24 \mathrm{~h}$ then repeated, adding the same amount of $\mathrm{KMnO}_{4}$ three further times. $24 \mathrm{~h}$ after the $4^{\text {th }}$ addition, the mixture was slowly poured into ice-water ( 600 $\mathrm{ml})$. Hydrogen peroxide $(6 \mathrm{ml}, 35 \%)$ was then added dropwise. The resulting viscous mixture was centrifuged to separate the oxidised graphite from the acid solvent. The resulting GO precipitate was washed with hydrochloric acid (5\%, $600 \mathrm{ml}$, Sigma-Aldrich) and centrifuged three times. The precipitate was then washed and centrifuged with distilled water $(600 \mathrm{ml})$ seven times until the $\mathrm{pH}$ of the supernatant liquid was $\approx 6$. The resulting dark brown viscous $\mathrm{GO}$ suspension was diluted to a concentration of $0.5 \mathrm{mg} \cdot \mathrm{ml}^{-1}$. The suspension was centrifuged with a Sorvall Legend XTR Centrifuge (Thermo Fisher Scientific, Waltham, MA, USA) at $3000 \mathrm{rpm}$ for 10 minutes to remove the unexfoliated graphite oxide.

The BP ink was prepared by sonicating crystalline black phosphorus powder (Smart Elements, Vienna, Austria) in acetonitrile (Sigma-Aldrich). An initial BP concentration of $5 \mathrm{mg}^{-1} \mathrm{ml}^{-1}$ was added to $15 \mathrm{~mL}$ acetonitrile, degassed, sealed with parafilm and sonicated for 24 hours using an Elmasonic $\mathrm{P} 70 \mathrm{H}$ ultrasonic bath (Elma
Schmidbauer, Singen, Germany) operated at $37 \mathrm{kHz}$ and $30 \%$ power. A home-made water cooling coil was used to prevent the reaction temperature exceeding $30{ }^{\circ} \mathrm{C}$ throughout the exfoliation process. After sonication, the dispersion was centrifuged using a 822 series benchtop centrifuge (Centurion Scientific, Chichester, UK) for $45 \mathrm{~min}$ at $1500 \mathrm{rpm}$. The top $10 \mathrm{ml}$ of the dispersion was collected for further analysis and inkjet printing.

\section{Inkjet printing GO and BP devices}

The fabrication process used with the GO and BP devices is shown schematically in Fig. 1. Interdigitated silver electrodes were printed onto the glass substrate (Fisherbrand) using a DMP 2800 printer (Fujifilm Dimatix, Santa Clara, CA, USA) equipped with a $10 \mathrm{pL}$ cartridge (DMC-11610), consisting of 16 independently addressable ejection nozzles with a diameter of around $21 \mu \mathrm{m}$ and distance of $254 \mu \mathrm{m}$ between adjacent nozzles. A silver nanoparticle ink (SigmaAldrich, Lot.\#MMKBT0133V) was filtered through a $0.45 \mu \mathrm{m}$ PTFE membrane prior to loading in the printer cartridge. Before printing, glass substrates were cleaned with ethanol and deionized water using an ultrasonic bath at $37 \mathrm{kHz}$ and $50 \%$ power for $15 \mathrm{~min}$, then dried with a pressurized $\mathrm{N}_{2}$ cleaning gun. During printing, the glass substrate was placed on the platen of the printer with its temperature set to $50^{\circ} \mathrm{C}$. Drop spacing was set to $30 \mu \mathrm{m}$ in the $\mathrm{x}$ - and $y$-directions. After printing the silver electrodes were annealed on a hot plate at $150{ }^{\circ} \mathrm{C}$ for $30 \mathrm{~min}$. to form a sintered high conductivity film.

The GO and BP sensor films were printed using an in-house designed and built laboratory scale inkjet printing platform (MPP 1000). This comprises an $x-y$ table (Micromech Systems, Braintree, UK), equipped with a piezoelectric actuated inkjet print head of internal diameter $60 \mu \mathrm{m}$ (MJ-ATP-01-60-8MX, Microfab Technologies Inc., Plano, TX, USA) with drive electronics (JetDrive III, Microfab) interfaced to a PC and controlled in a LabVIEW (National Instruments, Austin, TX, USA) environment. This printing system was used to deposit 2D materials because it has a larger nozzle diameter than the DMC-11610 printhead cartridge available on the DMP2800 printer, which was needed for printing the GO ink that comntained large flakes. All samples were printed on the silver electrode pattern with a drop spacing $100 \mu \mathrm{m}$. The devices were then dried in a tubular furnace in a flowing $\mathrm{N}_{2}(300 \mathrm{sccm})$ atmosphere, at $80^{\circ} \mathrm{C}$ for $1 \mathrm{~h}$. 


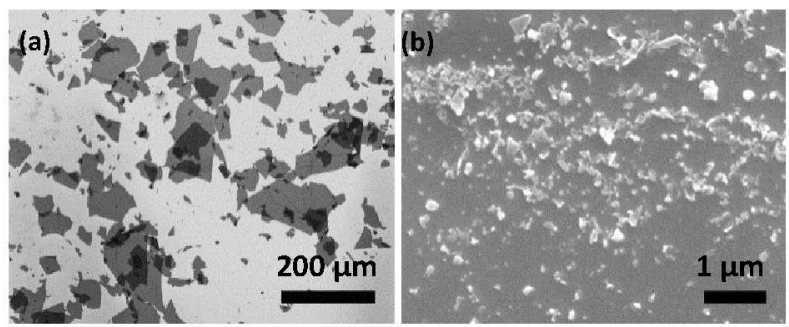

Fig. 2 SEM images of (a) GO sheets and (b) BP nanoflakes deposited on $\mathrm{Si} / \mathrm{SiO}_{2}$ substrates.

\section{Characterization methods}

Atomic force microscopy (AFM) images of the GO and BP nanosheets and the morphology of the films were obtained using a Dimension 3100 in tapping mode (Bruker, Billerica, MA, USA). Scanning electron microscopy (SEM) images were acquired using a XL30 field emission gun (FEG) electron microscope (FEI, Eindhoven, Netherlands) with a secondary electron detector. Raman spectra were obtained using a 2000 Raman spectrometer system (Renishaw, Wooton-under-Edge, UK) with a HeNe laser ( $633 \mathrm{~nm}$ excitation). The surface profile data was obtained using a ContourGT optical profiler (Bruker). X-ray photoelectron spectroscopy (XPS) was performed with a Kratos Axis Ultra X-ray photoelectron spectrometer meter (Kratos, Manchester, UK); curve fitting was accomplished by CasaXPS software.

\section{Sensor measurements}

The moisture sensing properties were measured by exposing the GO and $\mathrm{BP}$ devices to a range of controlled relative humidity ( $\mathrm{RH}$ ) levels. To set up different $\mathrm{RH}$ environments, saturated aqueous solutions of $\mathrm{LiCl}, \mathrm{KCH}_{3} \mathrm{COO}, \mathrm{CaCl}_{2}, \mathrm{~K}_{2} \mathrm{CO}_{3}, \mathrm{NaBr}, \mathrm{CuCl}_{2}, \mathrm{NaCl}, \mathrm{KCl}$ and $\mathrm{K}_{2} \mathrm{SO}_{4}$ were prepared (Sigma-Aldrich) and placed in airtight glass vessels at a temperature of $20{ }^{\circ} \mathrm{C}$, which yielded atmospheres with $\mathrm{RHs}$ of $11 \%$, $23 \%, 33 \%, 43 \%, 57 \%, 67 \%, 75 \%, 85 \%$, and $97 \%$, respectively. ${ }^{8,12}$ The relative humidity of the saturated aqueous solutions was measured using a humidity sensor (ST-321 Humidity Meter: Farnell Element 14, Leeds, UK). The capacitance response of the $\mathrm{GO}$ and BP sensors was measured using a Portable Electrochemical Interface \& Impedance Analyser (Ivium Technologies B.V., Eindhoven, Netherlands) over the frequency range $10 \mathrm{~Hz}-1 \mathrm{MHz}$, with a testing voltage of $0.5 \mathrm{~V}$, at a temperature of $20{ }^{\circ} \mathrm{C}$. The $\mathrm{AC}$ conductance measurements were carried out using a Frequency Response Analyzer LCR meter (PSM1735, Newtons 4th Ltd, Leicester, UK) at $100 \mathrm{~Hz}$ and $1 \mathrm{kHz}$ with a bias voltage of $0.5 \mathrm{~V}$.

\section{Results and discussions}

\section{Preparation and characterization of GO and BP inks}

The as-prepared GO flakes showed a mean diameter of $35.9 \pm 23.2$ $\mu \mathrm{m}$ (standard deviation) based on an SEM analysis of $>400$ flakes deposited onto a $\mathrm{Si} / \mathrm{SiO}_{2}$ substrate (Fig. 2a). More than $97 \%$ of the flakes are larger than $10 \mu \mathrm{m}$ and individual flakes with diameters up

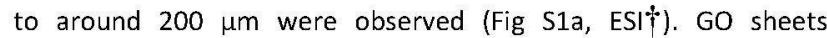
dispersed in water showed very good stability for more than one year due to the functional groups on the surface of the carbon. AFM measurements show the thickness of the GO sheets to be around 1 $\mathrm{nm}$, as shown in Fig.S1b and c (ESIF), indicating that the as-prepared GO sheets are monolayers. The Raman spectrum of the GO sheets (Fig S3a, ESI $\left.\right|^{\circ}$ ) showed typical D and G peaks at $1337 \mathrm{~cm}^{-1}$ and 1597 $\mathrm{cm}^{-1}$ respectively, which correspond to the reduction in size of the inplane $s p^{2}$ domains and the first-order scattering of the $E_{2 g}$ mode. ${ }^{37}$

The BP nanoflake dispersion was prepared by liquid phase exfoliation of BP crystals in solvents, similar to our previous work. ${ }^{38}$ Previous studies have found that the optimized solvents for exfoliating 2D layered materials should have a matched surface tension close to $40 \mathrm{~mJ} / \mathrm{m}^{2}$, such as $\mathrm{N}$-methylpyrrolidone (NMP) and dimethylformamide (DMF). ${ }^{39}$ However, these solvents have high boiling points $\left(>150^{\circ} \mathrm{C}\right.$ ) and need significant heat treatment to remove them, which is not appropriate for fabrication by inkjet printing. Hence, we chose acetonitrile for the exfoliation, which has a boiling point of $82{ }^{\circ} \mathrm{C}$ and has been previously reported as suitable for dispersing graphene. ${ }^{39}$ The resulting brown BP ink, as shown in Fig.1a, was found to be stable for several weeks. Any minor sedimentation could be readily be redispersed by slight shaking. Following the exfoliation process, the BP nanoflakes were drop cast on $\mathrm{Si} / \mathrm{SiO}_{2}$ substrates for SEM, AFM and Raman spectrum analysis. SEM images (Fig. 2b) shows that the size of individual BP nanoflakes ranges from tens of $\mathrm{nm}$ to about one $\mu \mathrm{m}$. AFM images (Fig. S2, ESI $\uparrow$ ) found the mean thickness of the BP nanoflakes to be around $8.5 \mathrm{~nm}$, which corresponds of approximately 10 atomic layers, assuming the layer spacing between mono-layer BP is $0.9 \mathrm{~nm}$. ${ }^{40,} 41$ The Raman spectrum shows the $A_{g}^{1}$ (out-of-plane mode), $B_{2 g}$ and $A_{g}^{2}$ (in-plane modes) peaks for BP nanoflakes are $\sim 362, \sim 439$, and $\sim 466 \mathrm{~cm}^{-1}$, respectively (Fig. S3b, ESI $\dagger$ ); these are consistent with data from crystalline and mechanically exfoliated BP flakes. ${ }^{42}$

\section{Morphology of the sensor devices}

The sensor device was fabricated by inkjet printing the GO $\sim \sim 0.5$ $\mathrm{mg} / \mathrm{ml})$ and $\mathrm{BP}(\sim 0.67 \mathrm{mg} / \mathrm{ml})$ colloidal inks onto an interdigitated pair of silver electrodes, previously deposited by inkjet printing, as shown in Figure $1 \mathrm{~b}$. The printed $\mathrm{Ag}$ electrode has a width of approximately $120 \mu \mathrm{m}$ and a thickness of about $210 \mathrm{~nm}$ (Fig. S4, ESI $\uparrow$ ). The measured conductivity of printed Ag electrode after sintering is $\sim 1.47 \times 10^{7} \mathrm{~S} \mathrm{~m}^{-1}$, which is about $23 \%$ of the value for bulk silver $\left(6.31 \times 10^{7} \mathrm{~S} \mathrm{~m}^{-1}\right)$. Fig. 3 shows SEM images of the printed GO and BP films on the sliver electrodes. It can be seen that the GO film is less wrinkled and homogeneous on the substrate and the silver electrodes (Fig. 3a-b), while the surface of the BP film is rough with a porous morphology (Fig. 3c-d). Droplets of the dried GO ink show a uniform morphology, while the dried BP droplets form a characteristic ring-like structure (Fig. S5, ESI $\uparrow$ ). The non-uniformity of the BP film is attributed to the coffee-ring that forms with the BP inks during solvent evaporation, which is a common phenomenon observed with inks containing 2D flakes. AFM measurements show the thickness of the GO film to be around $233 \mathrm{~nm}$, with a roughness of $12 \mathrm{~nm}$. The thickness of the BP film was determined to be approximately $640 \mathrm{~nm}$ with a roughness of $153 \mathrm{~nm}$ (Fig. S6, ESI †).

\section{Performance of the GO and BP sensors}

To investigate the performance of the GO and BP sensor devices, we measured their electrical properties under various levels of $\mathrm{RH}$. The humidity sensing experimental set up is shown in Fig. S7 (ESI †े). 


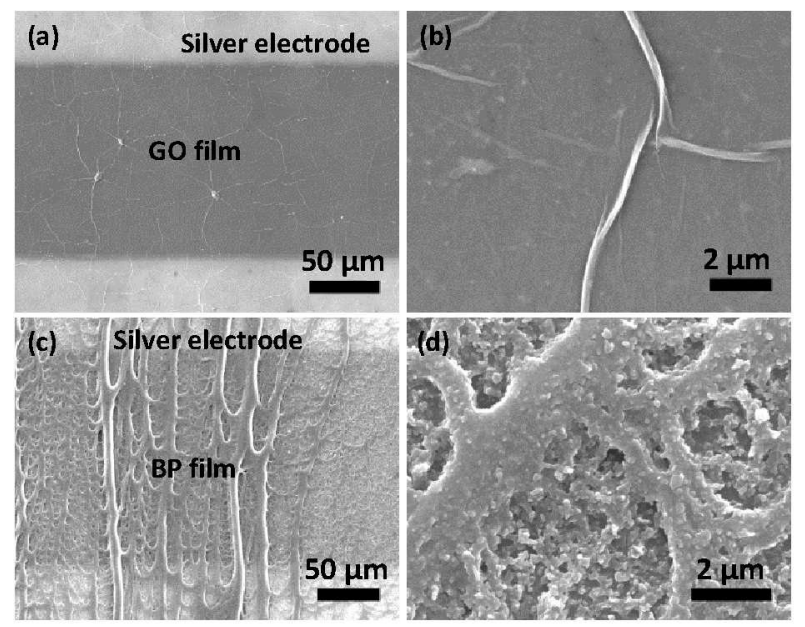

Fig. 3 SEM images of printed GO (a) and BP (c) films on the top of silver electrodes. High magnification micrograph of $G O(b)$ and $B P(d)$ films.

Complex impedance plots were measured over the frequency range $10 \mathrm{~Hz}-1 \mathrm{MHz}$, with a bias voltage of $0.5 \mathrm{~V}$ and at a temperature of $20^{\circ} \mathrm{C}$. As shown in Fig. 4, the impedance spectrum obtained from the $\mathrm{GO}$ and BP devices is very sensitive to the RH levels of the environment. The complex impedance spectrum changes from a semicircle to sickle-shaped when the humidity level increased from $11 \%$ RH to $97 \%$ RH (Fig. 3a-b). The impedance spectrum of the BP devices can be modelled by the equivalent circuits shown Fig. 4c (low humidity: $11 \%-43 \% \mathrm{RH}$, high humidity: $53 \%-97 \% \mathrm{RH}$ ). Here $R_{c t}$ represents the charge transfer resistance, CPE1 and CPE2 represent constant phase elements, which are used to model the film impedance and electrode/sensing film interface impedance, respectively. The impedance of a constant phase element $Z_{C P E}$ is generally defined as ${ }^{23}$

$$
Z_{C P E}=Q^{-1}(i \omega)^{-n}
$$

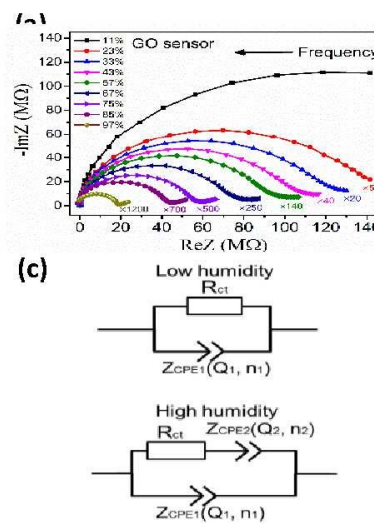

(b)

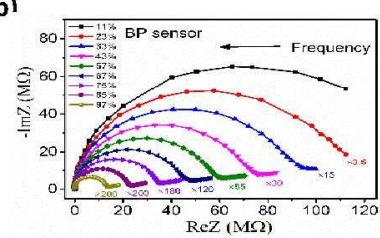

(d)

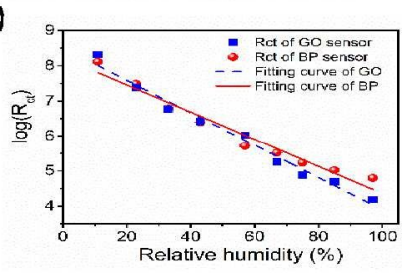

Fig. 4 Nyquist plots of the impedance of GO sensor (a) and BP sensor (b) under different humidity levels within in the frequency range $10 \mathrm{~Hz}$ to 1 MHz. ImZ: imaginary part; ReZ: real part. (c) Equivalent circuits of the sensor films under low humidity and high humidity. (d) Exponential behaviour of the $R_{c t}$ parameter vs different humidity levels for the $\mathrm{GO}$ and $\mathrm{BP}$ sensors.
Where $Q$ is a real parameter, $i$ the imaginary unit, $\omega$ the frequency, and $n$ a real parameter that can vary from 0 (pure resistor) to 1 (pure capacitor). The summary of the values of of $R_{c t}, n_{1}$, and $n_{2}$ obtained by fitting our data to the model are shown in Table $S 1$ (ESI + ). In the case of our sensor devices, the value of $n_{1}$ was always very close to 1 , which means $Z_{C P E 1}$ acts as an almost pure capacitor at all humidity levels. In addition, we observed that the dependence of $R_{c t}$ on RH can be approximated by an exponential relationship (Fig.4d).

For the GO sensor, the dependence of the $R_{c t}$ data can be expressed by $\log \left(R_{c t}\right)=-0.462 R H+8.5137$, with a linear regression coefficient of $R^{2}=0.9792$, while for the BP sensor device, the $R_{c t}$ data of the can be expressed as $\log \left(R_{c t}\right)=-0.0388 R H+8.2339$, with a linear regression coefficient of $R^{2}=0.9567$. The dependence of $R_{c t}$ on humidity in the GO film is from the presence of oxygenated functional groups in the basal plane and edges of the GO sheets, which could form a pathway for proton hopping sites when exposed to a humid environment. ${ }^{45}$ The humidity dependence of $R_{c t}$ in the BP film may be controlled by existing $\mathrm{PO}_{\mathrm{x}}$ functional groups or vacancy edges in the $\mathrm{BP}$ nanoflakes, which can be formed by the reaction of $\mathrm{H}_{2} \mathrm{O}$ or $\mathrm{O}_{2}$ during solvent exfoliation and the printing process. ${ }^{31}$ Protons generated via the adsorption of water molecules with active vacancies on the BP nanoflakes surface can cause a decrease in the electrical impedance.

Previous studies have proposed that the humidity sensing behaviour of materials is due to the dynamics of the proton tunnelling between adjacent water molecules via hydrogen bonds, i.e. the Grotthuss mechanism. ${ }^{3,46}$ In the case of the GO and BP sensors, water molecules are primarily chemisorbed onto the hydrophilic groups on the GO surface and active vacancies of the BP nanoflakes, forming a chemically-adsorbed water layer. Subsequently, another water molecule is physisorbed on the two adjacent hydroxyl groups through hydrogen bonding, forming a first physically-adsorbed water layer. As the humidity level increases, additional water molecules will physisorb on the top of the first physically-adsorbed water layer, forming a second physicallyadsorbed water layer. In this regime, water molecules are able to move freely inside the condensed water, which causes the proton conducting behaviour to become dominant (Grotthuss mechanism). ${ }^{3}$ It is this interaction of GO sheets and BP nanoflakes with water molecules that allows the $\mathrm{GO}$ and $\mathrm{BP}$ devices to be employed as humidity sensors.

To further investigate the performance of GO and BP devices as humidity sensors, we measured the response capacitance of the GO and $\mathrm{BP}$ sensors under different RH levels. Fig. $5 a-b$ shows that both the $\mathrm{GO}$ and $\mathrm{BP}$ sensors are extremely sensitive to both $\mathrm{RH}$ and frequency. The response capacitance of the GO sensor increases by almost four orders of magnitude as the RH levels increases from $11 \%$ to $97 \% \mathrm{RH}$, while the capacitance of the BP film increases almost three orders of magnitude over the same range of $\mathrm{RH}$. The increased capacitance can be attributed to more extensive adsorption of water molecules, which strengthens the polarization and increases the dielectric constant of the GO and BP films. ${ }^{8}$ To quantitatively describe humidity sensor sensitivity, the sensitivity of the device can be defined as 47

$$
\text { Sensitivity }=\left(C_{x}-C_{11}\right) / C_{11}
$$


(a)

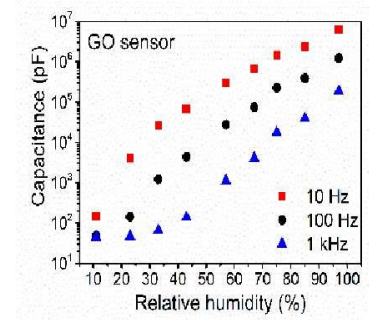

(c)

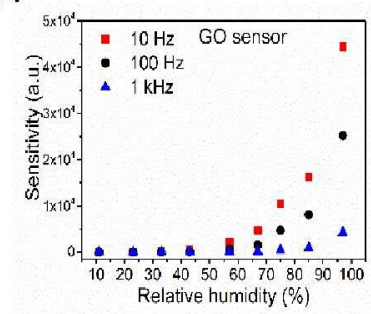

(b)

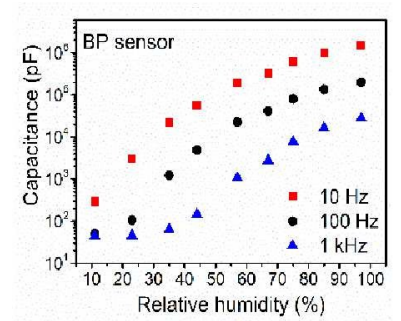

(d)

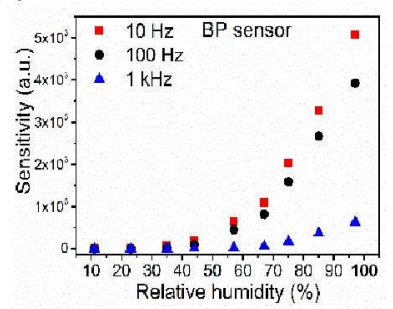

Fig. 5 Response capacitance (a, b) and sensitivity (c, d) of the GO and $\mathrm{BP}$ sensors as a function of RH levels at operation frequency of $10 \mathrm{~Hz}$, $100 \mathrm{~Hz}$, and $1 \mathrm{kHz}$.

where $C_{x}$ is the capacitance at $x \% \mathrm{RH}$ and $C_{11}$ is the capacitance at $11 \% \mathrm{RH}$.

Fig. 5c-d shows the relationship between sensitivity and $\mathrm{RH}$ defined by equation (2) at frequencies of $10 \mathrm{~Hz}, 100 \mathrm{~Hz}$, and $1 \mathrm{kHz}$ respectively. The $\mathrm{GO}$ and $\mathrm{BP}$ sensors both display a low capacitance $\left(\mathrm{C}_{\mathrm{GO}}=143.5 \mathrm{pF}, \mathrm{C}_{\mathrm{BP}}=297.7 \mathrm{pF}\right.$ ) at $11 \% \mathrm{RH}$ with frequency $10 \mathrm{~Hz}$. However, when the humidity increases to $97 \% \mathrm{RH}$, the capacitance of the $\mathrm{GO}$ and $\mathrm{BP}$ sensors rise to $6.38 \times 10^{6} \mathrm{pF}$ and $1.51 \times 10^{6} \mathrm{pF}$. This indicates a corresponding relative capacitance sensitivity of 72.6 $\mathrm{nF} / \% \mathrm{RH}$, and $17.6 \mathrm{nF} / \% \mathrm{RH}$ respectively. On increasing the operating frequency to $100 \mathrm{~Hz}$ and $1 \mathrm{kHz}$, the capacitance of the $\mathrm{GO}$ sensor changes by factors of $2.52 \times 10^{4}$ and $4.20 \times 10^{3}$, respectively. Whereas for the BP sensor operating at frequencies $100 \mathrm{~Hz}$ and $1 \mathrm{kHz}$ the capacitance changes by factors of $3.92 \times 10^{3}$ and $6.29 \times 10^{2}$, respectively. These values are much higher than the humidity sensing performance of $\mathrm{GO}$ alone, as well as for other 2D materials reported previously. ${ }^{8,17,48}$ Moreover, the GO and BP sensors also show high capacitance-sensitivity at low RH levels (less than $45 \% \mathrm{RH}$ ), which is appropriate for low-humidity sensing applications (Fig.S8, ESI $\dagger$ ). Although the largest capacitance change occurs at a relatively low frequency of $10 \mathrm{~Hz}$, the capacitance at low humidity increased during the cycle test at low frequency (Fig. S9, ESI $)$ ). This is due to the spacecharge polarization of adsorbed water when the electrical field direction changes slowly at low frequency; ${ }^{7}$ we therefore selected $100 \mathrm{~Hz}$ as the operating frequency for subsequent experiments.

Both response time and recovery time are important parameters for evaluating the effectiveness of humidity sensors. The response time is defined as the time required for the sensor capacitance to rise from its initial value to $90 \%$ of its final value with a $\mathrm{RH}$ change from $11 \% \mathrm{RH}$ to $97 \% \mathrm{RH}$ and the same for the recovery time when the $\mathrm{RH}$ is reduced from $97 \% \mathrm{RH}$ to $11 \% \mathrm{RH}$. Here, the response and recovery property of the humidity sensor were measured by alternately transferring the BP sensor between low (11\% RH) and high ( $97 \% \mathrm{RH})$

(a)

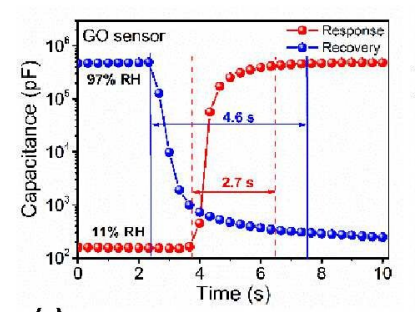

(c)

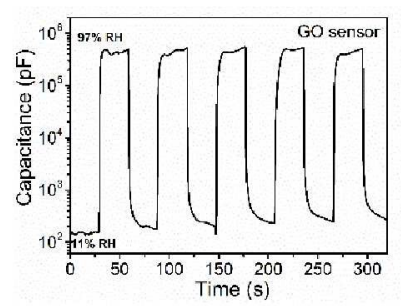

(b)

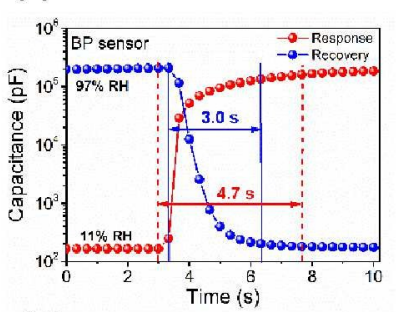

(d)

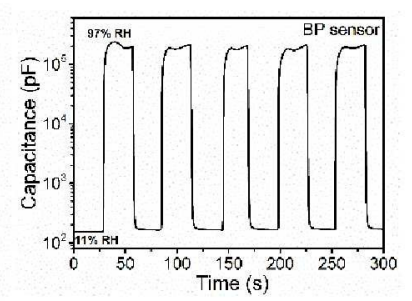

Fig. 6 The capacitance response and recovery behaviour of the GO (a) and BP (b) humidity sensors at humidity level between $11 \%$ and $97 \%$. Timeresolved response behaviour of the $\mathrm{GO}$ (c) and $\mathrm{BP}$ (d) humidity sensors under different humidity levels by changing humidity between $11 \% \mathrm{RH}$ and $97 \% \mathrm{RH}$ for 5 cycles. All measurements were conducted at $100 \mathrm{~Hz}$ and with a bias voltage of $0.5 \mathrm{~V}$.

humidity environments. Fig. $6 a$ and b show the typical capacitance response and recovery characteristic curves for the $\mathrm{GO}$ and $\mathrm{BP}$ humidity sensors. The $\mathrm{GO}$ sensor exhibits a fast response time of 2.7 $s$ and recovery time of $4.6 \mathrm{~s}$, whereas the BP sensor shows a response time of $4.7 \mathrm{~s}$ and recovery time of $3.0 \mathrm{~s}$. Both the response and recovery time of the $\mathrm{GO}$ and $\mathrm{BP}$ sensors are superior to conventional capacitive sensors based on anodic aluminium oxide ${ }^{47}$ and multi-wall carbon nanotubes. ${ }^{49}$ In addition, the GO and BP sensors also show excellent repeatability after several cycles (Fig $6 \mathrm{c}$ and $\mathrm{d}$ ).

To further investigate the response and recovery property of the sensor under a range of $\mathrm{RH}$ conditions, the BP sensor was sequentially transferred from ambient humidity (about $36 \% \mathrm{RH}$ ) to $11 \%, 23 \%, 33 \%, 43 \%, 57 \%, 67 \%, 75 \%, 85 \%$, and $97 \% \mathrm{RH}$. In all cases, the $\mathrm{BP}$ humidity sensor showed a very sensitive and rapid response for all RH levels (Fig. S10, ESI†). An apparent capacitance difference was found even when the RH difference is as little as 3\% (from $36 \%$ to $33 \% \mathrm{RH}$ ), which indicates that the BP device can be used for sensing small changes in $\mathrm{RH}$. Moreover, the capacitance of $\mathrm{GO}$ and BP sensors showed no discernible change when the samples were measured in a fixed humidity condition (11\% RH) for about 24 hours (Fig. S11, ESI $\nrightarrow$ ). This is consistent with previous reports of the stability of $\mathrm{GO}^{8,17}$ and $\mathrm{BP}{ }^{30}$ sensors.

Table 1 shows a comparison of the GO and BP sensors reported in this work with previously reported devices that have used 2D materials as the sensing component. Both the response time and recovery time of the sensors described here are superior to those previously reported for GO and semiconducting TMDCs sensors. Graphene-based sensors show the fastest response time $(0.25 \mathrm{~s})$ and recovery time $(0.35 \mathrm{~s})$. However, the sensitivity of graphene sensors is lower than most other sensors. Our GO sensor displays the highest sensitivity, while the sensitivity of the BP sensor is still better than most of the other sensors reported in Table 1. A possible mechanism 
Table 1 Comparison of reported humidity sensors using 2D materials as the sensing layer

\begin{tabular}{|c|c|c|c|c|c|c|}
\hline Sensing materials & $\begin{array}{l}\mathrm{RH} \text { range } \\
(\%)\end{array}$ & Sensing principle & $\begin{array}{l}\text { Response time } \\
\text { (s) }\end{array}$ & $\begin{array}{l}\text { Recovery time } \\
\text { (s) }\end{array}$ & $\begin{array}{l}\text { Sensitivity } \\
(\%)\end{array}$ & Ref. \\
\hline $\mathrm{GO}$ & $10-98$ & Piezoelectric & 19 & 10 & $79.3(\Delta \mathrm{V} / \% \mathrm{RH})$ & 4 \\
\hline GO & $15-95$ & Capacitive & 10.5 & 41 & $37757(\Delta \mathrm{C} / \mathrm{C})$ & 8 \\
\hline GO & $40-88$ & Resistive & $\sim 5$ & $\sim 6$ & $\sim 120000(\Delta \mathrm{l} / \mathrm{l})$ & 16 \\
\hline $\mathrm{GO} /$ polyelectrolyte & $11-97$ & Capacitive & - & - & $265640(\Delta C / C)$ & 17 \\
\hline rGO & $30-90$ & Resistive & 28 & 48 & $0.0423(\log Z / \% \mathrm{RH})$ & 18 \\
\hline Graphene & $1-96$ & Resistive & 0.6 & 0.4 & $0.31(\Delta \mathrm{R} / \mathrm{R} \% \Delta \mathrm{RH})$ & 19 \\
\hline \multirow{2}{*}{$\begin{array}{l}\text { Graphene/ } \\
\text { methyl-red }\end{array}$} & $5-95$ & Resistive & 0.25 & 0.35 & $93.36(\Delta R / R)$ & \multirow{2}{*}{21} \\
\hline & $5-95$ & Capacitive & - & - & $2869500(\Delta \mathrm{C} / \mathrm{C})$ & \\
\hline $\mathrm{VS}_{2}$ & $0-100$ & Resistive & $30-40$ & $12-50$ & $30(\Delta R / R)$ & 22 \\
\hline $\operatorname{Mos}_{2}$ & $0-60$ & Resistive & 9 & 17 & $\sim 150(\Delta R / R)$ & 24 \\
\hline $\mathrm{WS}_{2}$ & $40-80$ & Resistive & 13 & 17 & $3750(\Delta R / R)$ & 25 \\
\hline $\mathrm{BP}$ & $11-97$ & Resistive & 255 & 10 & $99.17(\Delta R / R)$ & 27 \\
\hline GO & $11-97$ & Capacitive & 2.7 & 4.6 & $4450544(\Delta \mathrm{C} / \mathrm{C})$ & This \\
\hline BP & $11-97$ & Capacitive & 4.7 & 3.0 & $507825(\Delta \mathrm{C} / \mathrm{C})$ & work \\
\hline
\end{tabular}

for the high performance of our GO sensor is the large size of the GO sheets used (mean lateral size of $\sim 36 \mu \mathrm{m}$ and considerably larger single flakes observed, as shown in Fig. 2a). Previous studies indicated that a decrease in the GO sheet size increased the number of sheet-to-sheet junction or boundaries, resulting in a higher amount of carboxylic acid groups, which tended to block the proton transport pathway and decreasing the sensitivity. ${ }^{45}$ Until recently it was not believed possible to print such large size GO flakes. ${ }^{36}$

GO film contains various oxygenated functional groups (Fig. S12a, ESI + ), which makes it strongly hydrophilic. Previous work has suggested that the epoxide and hydroxyl groups on the basal plane of the $\mathrm{GO}$ sheets form networks for efficient proton transfer through the Grotthuss mechanism: $\mathrm{H}_{2} \mathrm{O}+\mathrm{H}_{3} \mathrm{O}^{+} \rightarrow \mathrm{H}_{3} \mathrm{O}^{+}+\mathrm{H}_{2} \mathrm{O} .{ }^{45}$ The lower sensitivity to humidity seen with sensors based on $\mathrm{rGO}$, graphene, $V S_{2}, M_{2}$, and $W S_{2}$ listed in Table 1, is thus due to the lack of appropriate defects and functional groups capable of mediating proton transfer by this mechanism. Although the pristine BP does not contain epoxide and hydroxyl groups, it is well known that BP nanoflakes react with oxygen and/or water during the exfoliation process to form phosphorus oxide (POx) on the edges of BP flakes
(Fig. S12b, ESI $)$ ). We hypothesise that these functionalities allow the BP nanoflakes to easily bond with water molecules and provide rapid proton conduction pathways.

We have also observed that the ultrasensitive moisture responsiveness of the $\mathrm{GO}$ and $\mathrm{BP}$ sensors allows them to be used them as contactless switches, as shown in Fig. 7a. The sensors display a rapid real-time change of capacitance responding to finger humidity after placing a finger approximately. $7 \mathrm{~mm}$ above the device at an operating frequency of $100 \mathrm{~Hz}$ (Fig $7 \mathrm{~b}$ and c). The capacitance of the $\mathrm{GO}$ and $\mathrm{BP}$ sensors shows more than one order of magnitude change as a function of fingertip proximity under ambient air conditions. The ultrasensitive performance of the $\mathrm{GO}$ and $\mathrm{BP}$ sensors allows the capture of fine features due to moisture modulation on the near surface of human fingertip..$^{22}$ If the finger is wrapped in impermeable tape, the sensor shows no response to the presence of the finger, indicating that it is responding to local humidity. The results of sensitivity for fingertip sensing based on the GO and BP sensors used in this work are comparable to that of previously reported GO sensors ${ }^{45}$ and compare very well with other sensors (a)

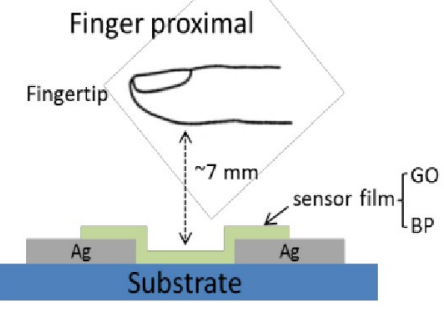

(b)

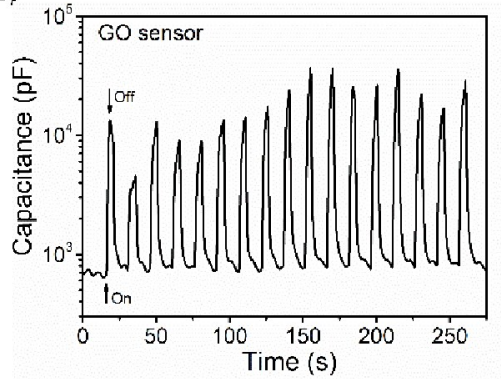

(c)

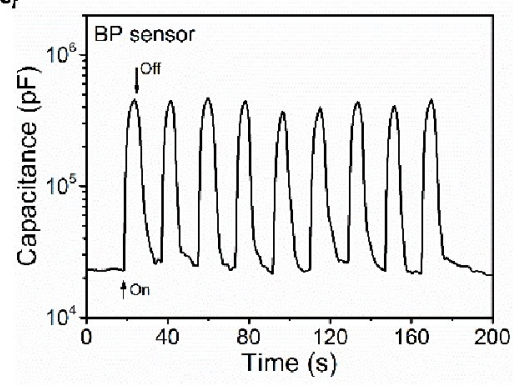

Fig. 7 (a) Experimental setup for contactless sensing of a proximal human fingertip. Time-resolved dynamic tests of the sensing response to a proximal human fingertip under ambient air conditions, with 'on' and 'off' states defined, (b) GO and (c) BP sensors. 
based on 2D materials, such as vanadium disulfide $\left(\mathrm{VS}_{2}\right)^{22}$ and reduced graphene oxide ( $\mathrm{rGO}) .{ }^{50}$

\section{Conclusions}

In summary, we have presented fully inkjet-printed, ultra-sensitive humidity sensors with fast dynamic response, using 2D layered structures of either GO or BP as sensing materials combined with silver nanoparticle electrodes. As the relative humidity level increased from 11 to $97 \%$, the response capacitance of the $\mathrm{GO}$ and BP sensors increased by four orders of magnitude at $10 \mathrm{~Hz}$ frequency, which show ultrahigh capacitive sensitivity factors of $4.45 \times 10^{4}$ and $5.08 \times 10^{3}$ respectively. Moreover, the measured response and recovery time of both the $\mathrm{GO}$ and $\mathrm{BP}$ sensors are within a few seconds. The GO and BP sensors show a rapid dynamic response to fingertip proximity, which provides a new concept for contactless switching. Overall, this work has demonstrated a low cost and scalable method for producing of high performance humidity sensors for printed electronics.

Conflict of Interest: The authors declare no competing financial interest.

\section{Acknowledgements}

The inkjet printing equipment used in this study was supported through EPSRC grant "Challenges in High Resolution Inkjet Printing" EP/L012022/1. PH would like to acknowledge the China Scholarship Council and the University of Manchester for financial support. $\mathrm{POB}$ and JB thank the Parker family for funding.

\section{References}

1 H. Farahani, R. Wagiran and M. N. Hamidon, Sensors, 2014, 14, 7881-7939.

2 K. M. Willett, N. P. Gillett, P. D. Jones and P. W. Thorne, Nature, 2007, 449, 710-716.

3 Z. Chen and C. Lu, Sens. Lett., 2005, 3, 274-295.

4 Y. Yao, X. D. Chen, H. H. Guo, Z. Q. Wu and X. Y. Li, Sens. Actuators, $B, 2012,161,1053-1058$.

5 V. K. Tomer, N. Thangaraj, S. Gahlot and K. Kailasam, Nanoscale, 2016, 8, 19794-19803.

6 Y. J. Guo, J. Zhang, C. Zhao, J. Y. Ma, H. F. Pang, P. A. Hu, F. Placido, D. Gibson, X. T. Zu, H. Y. Zu and Y. Q. Fu, Mater. Res. Bull., 2013, 48, 5058-5063.

7 B. C. Cheng, B. X. Tian, C. C. Xie, Y. H. Xiao and S. J. Lei, J. Mater. Chem., 2011, 21, 1907-1912.

8 H. Bi, K. Yin, X. Xie, J. Ji, S. Wan, L. Sun, M. Terrones and M. S. Dresselhaus, Sci. Rep., 2013, 3, 2714.

9 R. Demir, S. Okur and M. Seker, Ind. Eng. Chem. Res., 2012, 51, 3309-3313.

10 Y. L. Wang, X. C. Jiang and Y. N. Xia, J. Am. Chem. Soc., 2003, 125, 16176-16177.

11 Q. Kuang, C. S. Lao, Z. L. Wang, Z. X. Xie and L. S. Zheng, J. Am. Chem. Soc., 2007, 129, 6070-6071.

12 Z. G. Zhao, X. W. Liu, W. P. Chen and T. Li, Sens. Actuators, A, 2011, 168, 10-13.
13 J. W. Han, B. Kim, J. Li and M. Meyyappan, J. Phys. Chem. C, 2012, 116, 22094-22097.

14 S. S. Varghese, S. H. Varghese, S. Swaminathan, K. K. Singh and V. Mittal, Electronics, 2015, 4, 651-687.

15 N. O. Weiss, H. L. Zhou, L. Liao, Y. Liu, S. Jiang, Y. Huang and X. F. Duan, Adv. Mater., 2012, 24, 5782-5825.

$16 \mathrm{~S}$. Ghosh, R. Ghosh, P. K. Guha and T. K. Bhattacharyya, Nanotechno, IEEE Trans. on, 2015, 14, 931-937.

17 D. Z. Zhang, J. Tong, B. K. Xia and Q. Z. Xue, Sens. Actuators, B, 2014, 203, 263-270.

18 P. G. Su and C. F. Chiou, Sens. Actuators, B, 2014, 200, 9-18.

19 A. D. Smith, K. Elgammal, F. Niklaus, A. Delin, A. C. Fischer, S. Vaziri, F. Forsberg, M. Rasander, H. Hugosson, L. Bergqvist, S. Schroder, S. Kataria, M. Ostling and M. C. Lemme, Nanoscale, 2015, 7, 19099-19109.

20 W. J. Yuan and G. Q. Shi, J. Mater. Chem. A, 2013, 1, 10078-10091. 21 S. Ali, A. Hassan, G. Hassan, J. Bae and C. H. Lee, Carbon, 2017, 112, 130-130.

22 J. Feng, L. L. Peng, C. Z. Wu, X. Sun, S. L. Hu, C. W. Lin, J. Dai, J. L. Yang and Y. Xie, Adv. Mater., 2012, 24, 1969-1974.

23 S. Borini, R. White, D. Wei, M. Astley, S. Haque, E. Spigone, N. Harris, J. Kivioja and T. Ryhanen, ACS Nano, 2013, 7, 11166-11173.

24 S. L. Zhang, H. Jung, J. S. Huh, J. B. Yu and W. C. Yang, J. Nanosci. Nanotechno., 2014, 14, 8518-8522.

25 R. K. Jha and P. K. Guha, Nanotechnology, 2016, 27, 475503.

26 S. Eigler and A. Hirsch, Angew. Chem. Int. Ed., 2014, 53, 77207738.

27 D. J. Late, Micropor. Mesopor. Mat., 2016, 225, 494-503.

28 K. Hatakeyama, M. R. Karim, C. Ogata, H. Tateishi, A. Funatsu, T. Taniguchi, M. Koinuma, S. Hayami and Y. Matsumoto, Angew. Chem. Int. Edit., 2014, 53, 6997-7000.

29 K. Toda R. Furue and S. Hayami, Analytica Chim. Acta, 2015, 878, 43-53.

30 P. Yasaei, A. Behranginia, T. Foroozan, M. Asadi, K. Kim, F. KhaliliAraghi and A. Salehi-Khojin, ACS Nano, 2015, 9, 9898-9905.

31 D. Hanlon, C. Backes, E. Doherty, C. S. Cucinotta, N. C. Berner, C. Boland, K. Lee, A. Harvey, P. Lynch, Z. Gholamvand, S. Zhang, K. Wang, G. Moynihan, A. Pokle, Q. M. Ramasse, N. McEvoy, W. J. Blau, J. Wang, G. Abellan, F. Hauke, A. Hirsch, S. Sanvito, D. D. O'Regan, G. S. Duesberg, V. Nicolosi and J. N. Coleman, Nat. Commun., 2015, 6, 8563.

32 F. Bonaccorso, A. Bartolotta, J. N. Coleman and C. Backes, Adv. Mater., 2016, 28, 6136-6166.

33 E. A. Lewis, J. R. Brent, B. Derby, S. J. Haigh and D. J. Lewis, Chem. Commun., 2017, 53, 1445-1458.

34 G. Hu, T. Albrow-Owen, X. Jin, A. Ali, Y. Hu, R. C. T. Howe, K. Shehzad, Z. Yang, X. Zhu, R. I. Woodward, T.-C. Wu, H. Jussila, J.B. Wu, P. Peng, P.-H. Tan, Z. Sun, E. J. R. Kelleher, M. Zhang, Y. Xu and T. Hasan, Nat Commun, 2017, 8, 278.

35 A. Dimiev, D. V. Kosynkin, L. B. Alemany, P. Chaguine and J. M. Tour, J. Am. Chem. Soc., 2012, 134, 2815-2822.

36 H. Pei and B. Derby, 2D Mater., 2017, 4, 021021.

37 S. Stankovich, D. A. Dikin, R. D. Piner, K. A. Kohlhaas, A. Kleinhammes, Y. Jia, Y. Wu, S. T. Nguyen and R. S. Ruoff, Carbon, 2007, 45, 1558-1565.

38 J. R. Brent, N. Savjani, E. A. Lewis, S. J. Haigh, D. J. Lewis and P. O'Brien, Chem. Commun., 2014, 50, 13338-13341.

39 J. N. Coleman, M. Lotya, A. O'Neill, S. D. Bergin, P. J. King, U. Khan, K. Young, A. Gaucher, S. De, R. J. Smith, I. V. Shvets, S. K. Arora, G. Stanton, H. Y. Kim, K. Lee, G. T. Kim, G. S. Duesberg, T. Hallam, J. J. Boland, J. J. Wang, J. F. Donegan, J. C. Grunlan, G. Moriarty, A. Shmeliov, R. J. Nicholls, J. M. Perkins, E. M. Grieveson, K. 
Theuwissen, D. W. McComb, P. D. Nellist and V. Nicolosi, Science, 2011, 331, 568-571.

40 H. Liu, A. T. Neal, Z. Zhu, Z. Luo, X. F. Xu, D. Tomanek and P. D. D. Ye, ACS Nano, 2014, 8, 4033-4041.

41 P. Yasaei, B. Kumar, T. Foroozan, C. Wang, M. Asadi, D. Tuschel, J. E. Indacochea, R. F. Klie and A. Salehi-Khojin, Adv. Mater., 2015, 27, 1887-1892.

42 A. Avsar, I. J. Vera-Marun, J. Y. Tan, K. Watanabe, T. Taniguchi, A. H. C. Neto and B. Ozyilmaz, ACS Nano, 2015, 9, 4138-4145.

43 F. Torrisi, T. Hasan, W. P. Wu, Z. P. Sun, A. Lombardo, T. S. Kulmala, G. W. Hsieh, S. J. Jung, F. Bonaccorso, P. J. Paul, D. P. Chu and A. C. Ferrari, ACS Nano, 2012, 6, 2992-3006.

44 D. S. Eom, J. Chang, Y. W. Song, J. A. Lim, J. T. Han, H. Kim and K. Cho, J. Phys. Chem. C, 2014, 118, 27081-27090.
45 B.-H. Wee, W.-H. Khoh, A. K. Sarker, C.-H. Lee and J.-D. Hong, Nanoscale, 2015, 7, 17805-17811.

46 N. Agmon, Chem. Phys. Lett., 1995, 244, 456-462.

47 Y. Kim, B. Jung, H. Lee, H. Kim, K. Lee and H. Park, Sens. Actuators, $B, 2009,141,441-446$.

48 Z. Y. Zhang, J. D. Huang, Q. Yuan and B. Dong, Nanoscale, 2014, 6 , 9250-9256.

49 W. P. Chen, Z. G. Zhao, X. W. Liu, Z. X. Zhang and C. G. Suo, Sensors, 2009, 9, 7431-7444.

50 X. Wang, Z. Xiong, Z. Liu and T. Zhang, Adv. Mater., 2015, 27, 13701375. 


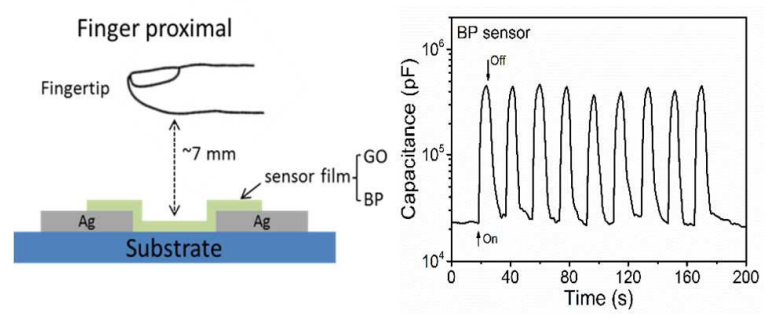

We present results from an all inkjet printed 2D-black phosphorous humidity sensor displaying very high sensitivity 